\title{
Nature of the constant factor in the relation between radial breathing mode frequency and tube diameter for single-wall carbon nanotubes
}

\author{
P. T. Araujo, ${ }^{1}$ I. O. Maciel, ${ }^{1}$ P. B. C. Pesce, ${ }^{1}$ M. A. Pimenta, ${ }^{1}$ S. K. Doorn, ${ }^{2}$ H. Qian, ${ }^{3}$ A. Hartschuh,,${ }^{3}$ M. Steiner, ${ }^{4}$ \\ L. Grigorian, ${ }^{5}$ K. Hata, ${ }^{6}$ and A. Jorio ${ }^{1,7}$ \\ ${ }^{1}$ Departamento de Física, Universidade Federal de Minas Gerais, Belo Horizonte, Minas Gerais 30123-970 Brazil \\ ${ }^{2}$ Chemistry Division, Los Alamos National Laboratory, Los Alamos, New Mexico 87545, USA \\ ${ }^{3}$ Departament Chemie und Biochemie and CeNS, Ludwig-Maxmilians-Universitaet Muenchen, Germany \\ ${ }^{4}$ IBM Research Division, T.J. Watson Research Center, Yorktown Heights, New York 10598, USA \\ ${ }^{5}$ YTC America Incorporated, Camarillo, California 93012, USA \\ ${ }^{6}$ Research Center for Advanced Carbon Materials, AIST, Tusukuba 305-8565, Japan \\ ${ }^{7}$ Divisão de Metrologia de Materiais, Instituto Nacional de Metrologia, Normalização e Qualidade Industrial (Inmetro), \\ Duque de Caxias, Rio de Janeiro 25250-020, Brazil \\ (Received 9 May 2008; published 25 June 2008)
}

\begin{abstract}
Resonance Raman scattering is used to determine the radial breathing mode (RBM) frequency $\left(\omega_{\mathrm{RBM}}\right)$ dependence on tube diameter $\left(d_{t}\right)$ for single-wall carbon nanotubes (SWNTs). We establish experimentally the $\omega_{\mathrm{RBM}}=227.0 / d_{t}$ as the fundamental relation for pristine SWNTs. All the other RBM values found in the literature can be explained by an upshift in frequency due mostly to van der Waals interaction between SWNTs and environment.
\end{abstract}

DOI: 10.1103/PhysRevB.77.241403

PACS number(s): 78.67.Ch, 71.35.-y, 73.22.-f, 78.30.Na

The radial breathing mode (RBM) provides the spectroscopic signature of single-wall carbon nanotubes (SWNTs). ${ }^{1,2}$ The RBM frequency $\left(\omega_{\mathrm{RBM}}\right)$ depends on the SWNT diameter $\left(d_{t}\right)$, which is related to their $(n, m)$ structural indices by $d_{t}=0.142 \sqrt{3\left(n^{2}+m n+m^{2}\right)} / \pi .^{2}$ The experimental results in the literature have been fitted with the relation $\omega_{\mathrm{RBM}}=\mathbf{A} / d_{t}+\mathbf{B}$, with values for $\mathbf{A}$ and $\mathbf{B}$ varying from paper to paper. ${ }^{3-12}$ The empirical constant factor $\mathbf{B}$ prevents the expected limit of a graphene sheet from being achieved, where the $\omega_{\mathrm{RBM}}$ should go to zero when $d_{t}$ approaches infinity. Therefore, $\mathbf{B}$ is supposedly associated with an environmental effect on $\omega_{\mathrm{RBM}}$, rather than an intrinsic property of SWNTs. Environmental effect here means the effect of the surrounding medium, such as bundling, molecules adsorbed from the air, surfactant used for SWNT bundle dispersion, and substrates where the tubes are sitting.

Here we use resonance Raman scattering to measure the RBMs of SWNTs grown by the water-assisted chemical vapor deposition (CVD) method. ${ }^{13-16}$ These SWNTs follow a simple linear relation between $\omega_{\mathrm{RBM}}$ and $d_{t}$, with the proportionality constant $\mathbf{A}=227.0 \mathrm{~cm}^{-1} \mathrm{~nm}$, in agreement with the elastic property of graphite, and with a negligible environmental effect $(\mathbf{B} \approx \mathbf{0})$. All the observed $\omega_{\mathrm{RBM}}$ reported in the literature are upshifted from this fundamental relation. ${ }^{3-12}$ The upshift exhibits a $d_{t}$ dependence in quantitative agreement with recent predictions considering the van der Waals interaction between SWNTs and environment. ${ }^{17}$

This water-assisted CVD process has been called "supergrowth" and generates millimeter-long isolated and highpurity SWNTs. ${ }^{13-16}$ The super-growth SWNTs exhibit a broad $d_{t}$ distribution $\left(d_{t}\right.$ from 1 to $\left.4 \mathrm{~nm}\right)$ and all tube chiralities $\left(0^{\circ} \leq \theta \leq 30^{\circ}\right)$. The SWNTs are vertically aligned from a silicon substrate to form a very sparse material, where SWNTs represent only $3.6 \%$ of the total volume. ${ }^{15}$ Two triple-monochromator Raman spectrometers (a Dilor XY in the visible and a SPEX in the near-infrared) with charge coupled device (CCD) detectors are used to acquire the spectra. In both cases a backscattering geometry is applied. An $\mathrm{Ar}-\mathrm{Kr}$ laser and two quasicontinuous (dye and Ti:sapphire) lasers are used to tune the excitation laser wavelength. The laser power density is maintained constant, low enough to avoid heating effects $(1 \mathrm{~mW}$ focused with an $80 \times$ objective in the visible, and $25 \mathrm{~mW}$ focused with a $10 \mathrm{~cm}$ focal length in the infrared).

In Fig. 1 we compared similar $\omega_{\text {RBM }}$ Raman spectra of two different samples. The gray lines show $\omega_{\mathrm{RBM}}$ spectra for the super-growth SWNTs and they are being compared to the black lines $\omega_{\mathrm{RBM}}$ spectra obtained from a SWNT sample grown by the alcohol-assisted CVD method. ${ }^{12}$ Looking at Figs. 1(a) and 1(b) it is clear that the super-growth $\omega_{\mathrm{RBM}}$ frequencies are downshifted from the $\omega_{\mathrm{RBM}}$ values for the other sample. The differences in the low frequency region

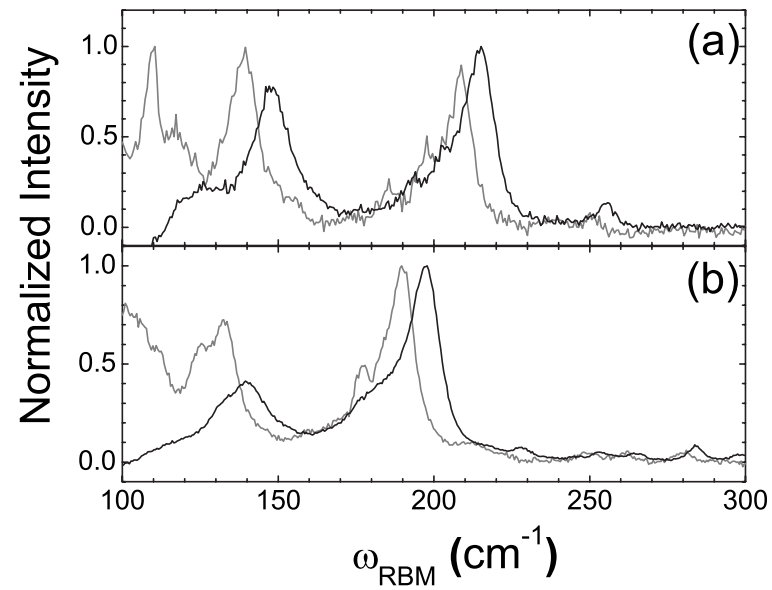

FIG. 1. The $\omega_{\text {RBM }}$ spectra for the super-growth SWNTs (gray) and for the "alcohol CVD" SWNTs (black). The spectra are obtained using different laser lines: (a) $590 \mathrm{~nm}$ (gray) and $600 \mathrm{~nm}$ (black); (b) $636 \mathrm{~nm}$ (gray) and $650 \mathrm{~nm}$ (black). 


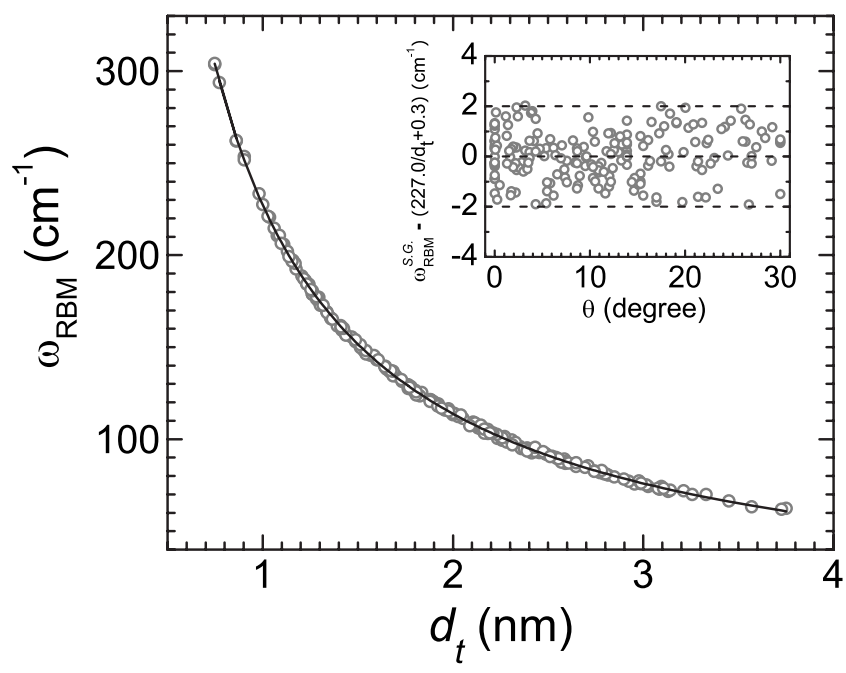

FIG. 2. Experimental radial breathing mode frequency $\left(\omega_{\mathrm{RBM}}\right)$ as a function of tube diameter $\left(d_{t}\right)$. The open circles represent the SWNT data assigned in this work and the solid line is a fitting to the data given by $\omega_{\mathrm{RBM}}=227.0 / d_{t}+0.3$. The inset plots the difference between the experimental $\omega_{\mathrm{RBM}}$ and $227.0 / d_{t}+0.3$ as a function of SWNT chiral angle $(\theta)$.

(below $\sim 120 \mathrm{~cm}^{-1}$ ) are due to different $d_{t}$ distributions in the samples.

With the super-growth sample, 125 Raman spectra, each at a different $E_{\text {laser }}$ excitation, are used to assign the $(n, m)$ structure of 197 different SWNTs (of which 73 are metallic and 124 are semiconducting). ${ }^{2}$ As a short explanation, each RBM spectrum exhibits bands that are composed of a convolution of Lorentzian peaks. Each Lorentzian corresponds to the RBM Stokes spectrum of one specific $(n, m)$ SWNT. The SWNTs observed are the ones having an optical transition energy (usually called $E_{i i}, i=1,2, \ldots$ ) in resonance with the excitation laser energy. The $(n, m)$ assignment is a complex but reliable procedure, and has already been extensively discussed in the literature (see Refs. 8-10 and especially Ref. 12). This analysis is important because all the $197(n, m)$ tubes assigned in this work have their optical transition energies $\left(E_{i i}\right)$ upshifted from others in the literature. ${ }^{5,6,8-10,12,18-21}$ To compare the $\omega_{\text {RBM }}$ for the same set of resonant $(n, m)$ SWNTs in the two samples, the two spectra in either Fig. 1(a) or Fig. 1(b) have to be obtained with different laser excitations (see caption to Fig. 1). While the alcohol-assisted CVD sample has a specific set of SWNT species in resonance at a given laser excitation energy, the super-growth sample has the same species resonant for a higher $(\Delta E \sim 40 \mathrm{meV})$ laser energy.

Using all the $197(n, m)$ SWNTs assigned and the relation $d_{t}=0.142 \sqrt{3\left(n^{2}+m n+m^{2}\right)} / \pi$, the experimental $\omega_{\mathrm{RBM}}$ can be plotted as a function of $d_{t}$, as shown in Fig. 2. Fitting the data shown in Fig. 2 using the relation $\omega_{\mathrm{RBM}}=\mathbf{A} / d_{t}+\mathbf{B}$ we obtain $\mathbf{A}=(\mathbf{2 2 7 . 0} \pm \mathbf{0 . 3}) \mathrm{cm}^{-1} \mathrm{~nm}$ and $\mathbf{B}=(\mathbf{0 . 3} \pm \mathbf{0 . 2}) \mathrm{cm}^{-1}$. This result is unexpected for being different from all results in the literature, ${ }^{3-12}$ and it is remarkable because a pristinelike relation between $\omega_{\mathrm{RBM}}$ and $d_{t}$ with a negligible environmental effect $(\mathbf{B} \approx \mathbf{0})$ has been obtained, so that $\omega_{\mathrm{RBM}} \rightarrow 0$ as $d_{t} \rightarrow \infty$. Furthermore, the value of the constant
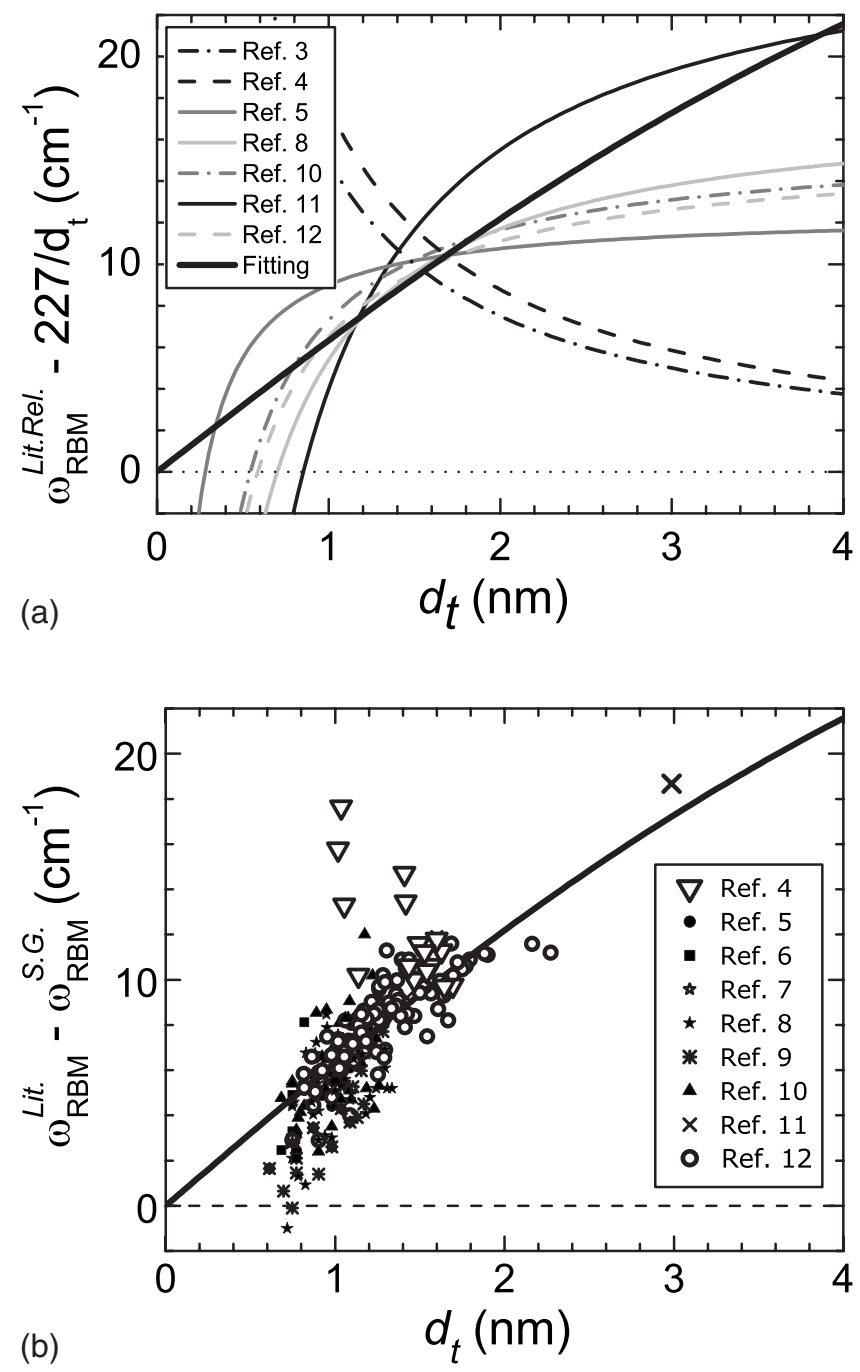

FIG. 3. (a) Difference between $\omega_{\mathrm{RBM}}$ relations from the literature $\left(\omega_{\mathrm{RBM}}^{\text {Lit.Rel. }}\right)$ and $\omega_{\mathrm{RBM}}=227.0 / d_{t}$ as a function of tube diameter $\left(d_{t}\right)$. (b) Difference between $\omega_{\mathrm{RBM}}$ data from the literature $\left(\omega_{\mathrm{RBM}}^{\text {Lit. }}\right)$ and $\omega_{\mathrm{RBM}}$ data from this work $\left(\omega_{\mathrm{RBM}}^{S . G .}\right)$ as a function of $d_{t}$. Each symbol represents data from a different reference (see legends). The thick solid line is a fit to the data in (b), as discussed in the text, and it is also shown in (a).

$\mathbf{A}=227.0 \mathrm{~cm}^{-1} \mathrm{~nm}$ that accounts for the vibrational characteristics of SWNTs exactly matches the value predicted by elasticity theory 22,23 and is in excellent accordance with the value predicted by recent tight-binding method calculations for isolated SWNTs. ${ }^{24}$ Both the theoretical methods are parameterized by the speed of sound in graphite. Therefore, this result directly connects one-dimensional carbon nanotubes and their two-dimensional counterpart graphene.

To understand why the $\omega_{\mathrm{RBM}}$ dependence with $d_{t}$ in supergrowth SWNTs is unique, the results obtained here are compared with the results in the literature. Figure 3(a) shows the difference between several $\omega_{\mathrm{RBM}}=\mathbf{A} / d_{t}+\mathbf{B}$ found in the literature ${ }^{3-5,8,10-12}$ and the $\omega_{\mathrm{RBM}}=227.0 / d_{t}$ established here. All the curves in the literature converge within the 1 to $2 \mathrm{~nm}$ $d_{t}$ range, that is where most of the experimental data were actually obtained. Figure 3(b) shows the difference between the $\omega_{\mathrm{RBM}}$ experimental values from the literature $\left(\omega_{\mathrm{RBM}}^{\mathrm{Lit.}}\right)$ 
(Refs. 4-12) and from this work ( $\omega_{\mathrm{RBM}}^{S . G}$, S.G. stands for super-growth) as a function of $d_{t}$. All the published results for $\omega_{\mathrm{RBM}}^{\mathrm{Lit}}$ are grouped in Fig. 3(b) on a $d_{t}$ dependent upshifted trend for $\Delta \omega_{\mathrm{RBM}}=\omega_{\mathrm{RBM}}^{\mathrm{Lit}}-\omega_{\mathrm{RBM}}^{S . G}$. The down-triangles in Fig. 3(b) that spread out from the general trend are related to the $\omega_{\mathrm{RBM}}=248 / d_{t}$ relation [dashed dark-gray curve from Ref. 4 in Fig. 3(a)] proposed in $2001^{4}$ by imposing the $\mathbf{B}=0$ condition on the basis of $\omega_{\mathrm{RBM}} \rightarrow 0$ as $d_{t} \rightarrow \infty$. The $\omega_{\mathrm{RBM}}=248 / d_{t}$ relation was obtained with an $E_{i i}$ vs $d_{t}$ relation that did not consider nanotube curvature effects (the firstneighbor tight-binding model), which are important for small $d_{t}$ tubes (below $d_{t}=1.2 \mathrm{~nm}$ ). ${ }^{2}$ Therefore, the scatter in the data from the general trend in Fig. 3(b) might be due to a wrong $(n, m)$ assignment below $d_{t}=1.2 \mathrm{~nm}$. It is likely that the same argument is valid for the $\omega_{\mathrm{RBM}}$ vs $d_{t}$ relation obtained in Ref. 3 [black dashed-dotted line in Fig. 3(a)], since it is also based on the first-neighbor tight-binding model. Therefore, we demonstrated here that the $d_{t}$ dependence of the difference between the experimental data in the literature and the fundamental relation $\omega_{\mathrm{RBM}}=227.0 / d_{t}$ is always the same.

A recent molecular dynamics calculation considering van der Waals interactions between SWNTs and a shell of adsorbed fluid ${ }^{17}$ is now applied to explain the result shown in Fig. 3(b). Longhurst and Quirke ${ }^{17}$ considered SWNTs within the 1.03 to $1.73 \mathrm{~nm}$ diameter range surrounded by water at $300 \mathrm{~K}$ and showed a $d_{t}$ dependent upshift on $\omega_{\mathrm{RBM}}$ as compared to the tube in vacuum, ranging from 4 to $10 \mathrm{~cm}^{-1}$, in perfect agreement with our observation in Fig. 3(b). The shifts were explained by the interaction of the RBM with the adsorbed shell of fluid surrounding the nanotube via Lennard-Jones potential, plus a small contribution of static pressure difference at the nanotube surface $(10 \%-20 \%$ of the total effect). Then they proposed a model that fits their calculations, considering the RBM of a coupled system SWNT/ environment composed of two spring constants: one coming from the $\mathrm{C}-\mathrm{C}$ bond strength and the other coming from the interaction strength between the SWNT and its surroundings. The problem of addressing the environmental effect on $\omega_{\mathrm{RBM}}$ is now reduced to solving a simple harmonic oscillator for a cylindrical shell subjected to an inwards pressure $[p(x)]$ given by

$$
\frac{2 x(t)}{d_{t}}+\frac{\rho h}{E h}\left(1-\nu^{2}\right) \frac{\partial^{2} x(t)}{\partial t^{2}}=-\frac{\left(1-\nu^{2}\right)}{E h} p(x),
$$

where $x(t)$ is the displacement in the radial direction, $p(x)$ $=\left(24 K / s_{0}^{2}\right) x(t), K$ (in $\left.\mathrm{eV} / \AA^{2}\right)$ gives the van der Waals interaction strength, $s_{0}$ is the equilibrium separation between the SWNT wall and the environment shell, $E$ is the Young's modulus, $\rho$ is the mass density per unit volume, $\nu$ is the Poisson's ratio, and $h$ represents the thickness of the shell. If $p(x)$ is null, Eq. (1) gives the fundamental $\omega_{\mathrm{RBM}}^{0}$ for a pristine SWNT,

$$
\omega_{\mathrm{RBM}}^{0}=\left\{\frac{1}{\pi c}\left[\frac{E h}{\rho h\left(1-\nu^{2}\right)}\right]^{1 / 2}\right\} \frac{1}{d_{t}},
$$

where the term inside the curly bracket is established here as $227.0 \mathrm{~cm}^{-1} \mathrm{~nm}$. For $p(x)$ non-null we have

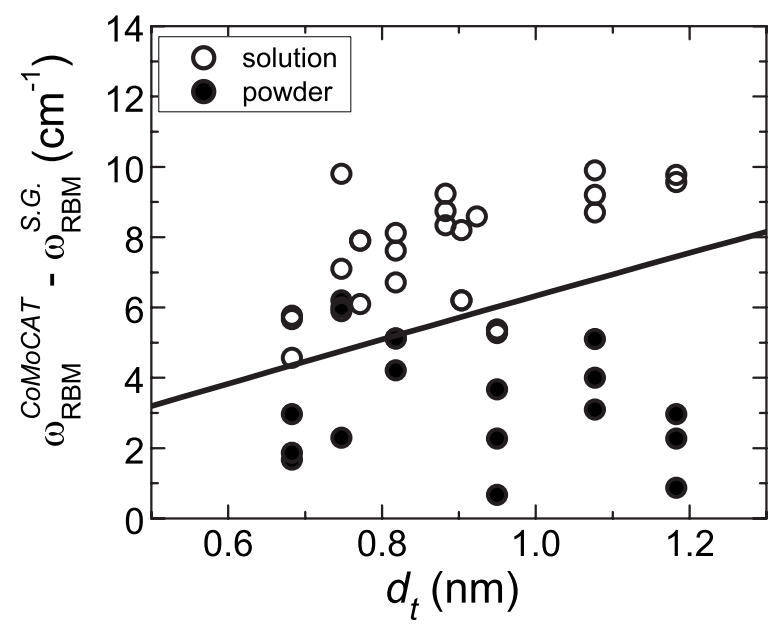

FIG. 4. Difference between CoMoCAT SWNT RBM frequencies $\left(\omega_{\mathrm{RBM}}^{\mathrm{CoMoCAT}}\right)$ and $\omega_{\mathrm{RBM}}^{S . G .}$ as a function of $d_{t}$. The open circles stand for SWNTs in solution and filled circles stand for SWNTs in powder. The thick solid line represents the fitting shown in Fig. 3(b).

$$
\omega_{\mathrm{RBM}}^{\prime}=227.0\left[\frac{1}{d_{t}^{2}}+\frac{6\left(1-\nu^{2}\right)}{E h} \frac{K}{s_{0}^{2}}\right]^{1 / 2},
$$

where $\left[6\left(1-\nu^{2}\right) / E h\right]=26.3 \AA^{2} / \mathrm{eV}$. The shift in $\omega_{\mathrm{RBM}}^{\prime}$ due to the environment is given by $\Delta \omega_{\mathrm{RBM}}=\omega_{\mathrm{RBM}}^{\prime}-\omega_{\mathrm{RBM}}^{0}$. The data in Fig. 3(b) are fitted (see black thick solid line) by considering $K / s_{0}^{2}$ in Eq. (3) as an adjustable parameter. The best fit is obtained with $K / s_{0}^{2}=(2.2 \pm 0.1) \mathrm{meV} / \AA^{4}$. The $d_{t}$ dependent behavior of the environmental effect in $\omega_{\mathrm{RBM}}$ is then established up to $d_{t}=3 \mathrm{~nm}$. A similar environmental effect is obtained for SWNTs in bundles, ${ }^{3,12}$ surrounded by different surfactants, ${ }^{5-10}$ in air suspended by posts ${ }^{11}$ or sitting on a $\mathrm{SiO}_{2}$ substrate, ${ }^{4}$ but absent in the super-growth SWNTs.

In fact, indication for environmental dependent effects is observed in the lower $d_{t}$ range, ${ }^{25}$ as shown by the large spread of data in Fig. 3(b) for $d_{t}<1.2 \mathrm{~nm}$. Notice that 1.2 $\mathrm{nm}$ is also the limit where curvature effects become important. ${ }^{2,26}$ Therefore, to complete our $\omega_{\mathrm{RBM}}$ analysis, Raman spectroscopy experiments in low $d_{t}$ CoMoCAT SWNT samples are performed in two different environments, (i) asgrown powder and (ii) dispersed in $\mathrm{D}_{2} \mathrm{O}$ using CTAB (cetyltrimethylammoniumbromine) as the surfactant. ${ }^{27}$ Figure 4 shows the result of the same analysis as presented in Fig. 3(b). Again, all the $\Delta \omega_{\mathrm{RBM}}$ are positive, going up to $+10 \mathrm{~cm}^{-1}$, and a clear environment dependence is observed. The $\omega_{\text {RBM }}$ for SWNTs in solution (open circles) are clearly upshifted from the $\omega_{\text {RBM }}$ for SWNTs in powder (full black circles). Three different sets of SWNTs in solution and powder were measured and the results were reproducible.

In summary, super-growth SWNTs exhibit a relation $\omega_{\mathrm{RBM}}=227.0 / d_{t}$ that gives the fundamental spectroscopic signature of the SWNT $d_{t}$. This relation is in agreement with calculations using the velocity of sound in graphite and has $\omega_{\mathrm{RBM}} \rightarrow 0$ as $d_{t} \rightarrow \infty$, recovering the $2 \mathrm{D}$ graphene. The energies $E_{i i}^{S . G .}$ measured in the super-growth SWNTs are generally blueshifted from the $E_{i i}^{\mathrm{Lit}}$ in the literature 
$(\Delta E \sim 40 \mathrm{meV})$, suggesting smaller environment dielectric constant (close to 1$)$. All the $\omega_{\mathrm{RBM}}$ results in the literature are upshifted from the pristine values due to van der Waals interaction with the environment, and can be generally described by

$$
\omega_{\mathrm{RBM}}^{\mathrm{Lit.}}=\frac{227}{d_{t}} \sqrt{1+C * d_{t}^{2}},
$$

where $C=\left[6\left(1-\nu^{2}\right) / E h\right]\left[K / s_{0}^{2}\right]=0.05786 \mathrm{~nm}^{-2}$. For $d_{t}$ $<1.2 \mathrm{~nm}$, where the curvature effects become important, the environment effect depends more critically on the specific sample, the observed environmental-induced upshifts ranging from 1 to $10 \mathrm{~cm}^{-1}$. The super-growth SWNTs discussed here are shielded from the environment by their own struc- ture, that is a dense forest. We propose that all SWNT property measurements, such as exciton binding energy, temperature, doping, pressure dependent responses, etc. be revisited with similar samples, to provide what could be a solid baseline for the properties of pristine SWNTs. Furthermore, having established the fundamental $d_{t}$ dependence, the $\omega_{\mathrm{RBM}}$ can now be used as a probe for understanding surface tension, wetting on an attractive substrate, and the interaction potential for different liquids with respect to graphite and carbon nanotubes. ${ }^{17}$

We acknowledge M. S. Dresselhaus and T. Gokus, and financial support from Rede Nacional de Pesquisa em Nanotubos de Carbono-MCT/CNPq (Brazil), LANL LDRD program (USA), and Capes-DAAD/PROBRAL.
${ }^{1}$ P. McEuen, in Introduction to Solid State Physics, edited by C. Kittel (Wiley, New York, 2005), Chap. 18.

${ }^{2}$ A. Jorio, M. S. Dresselhaus, and G. Dresselhaus, Carbon Nanotubes: Advanced Topics in Synthesis, Properties, and Applications, Topics in Applied Physics Vol. 111 (Springer, Berlin, 2008).

${ }^{3}$ M. Milnera, J. Kurti, M. Hulman, and H. Kuzmany, Phys. Rev. Lett. 84, 1324 (2000).

${ }^{4}$ A. Jorio, R. Saito, J. H. Hafner, C. M. Lieber, M. Hunter, T. McClure, G. Dresselhaus, and M. S. Dresselhaus, Phys. Rev. Lett. 86, 1118 (2001).

${ }^{5}$ S. M. Bachilo, M. S. Strano, C. Kittrell, R. H. Hauge, R. E. Smalley, and R. B. Weisman, Science 298, 2361 (2002).

${ }^{6}$ A. Hartschuh, H. N. Pedrosa, L. Novotny, and T. D. Krauss, Science 301, 1354 (2003)

${ }^{7}$ M. Strano, S. K. Doorn, E. H. Haroz, C. Kittrell, R. H. Hauge, and R. E. Smalley, Nano Lett. 3, 1091 (2003).

${ }^{8}$ H. Telg, J. Maultzsch, S. Reich, F. Hennrich, and C. Thomsen, Phys. Rev. Lett. 93, 177401 (2004).

${ }^{9}$ S. K. Doorn, D. A. Heller, P. W. Barone, M. L. Usrey, and M. S. Strano, Appl. Phys. A: Mater. Sci. Process. 78, 1147 (2004).

${ }^{10}$ C. Fantini, A. Jorio, M. Souza, M. S. Strano, M. S. Dresselhaus, and M. A. Pimenta, Phys. Rev. Lett. 93, 147406 (2004).

${ }^{11}$ M. Paillet, T. Michel, J. C. Meyer, V. N. Popov, L. Henrard, S. Roth, and J. L. Sauvajol, Phys. Rev. Lett. 96, 257401 (2006).

${ }^{12}$ P. T. Araujo, S. K. Doorn, S. Kilina, S. Tretiak, E. Einarsson, S. Maruyama, H. Chacham, M. A. Pimenta, and A. Jorio, Phys. Rev. Lett. 98, 067401 (2007).

${ }^{13}$ K. Hata, D. N. Futaba, K. Mizuno, T. Namai, M. Yumura, and S. Iijima, Science 306, 1362 (2004).
${ }^{14}$ D. N. Futaba, K. Hata, T. Yamada, K. Mizuno, M. Yumura, and S. Iijima, Phys. Rev. Lett. 95, 056104 (2005).

${ }^{15}$ D. N. Futaba, K. Hata, T. Namai, T. Yamada, K. Mizuno, Y. Hayamizu, M. Yumura, and S. Iijima, J. Phys. Chem. B 110, 8035 (2006).

${ }^{16}$ D. N. Futaba, K. Hata, T. Yamada, T. Hiraoka, Y. Hayamizu, Y. Kakudate, O. Tanaike, H. Hatori, M. Yumura, and S. Iijima, Nat. Mater. 5, 987 (2006).

${ }^{17}$ M. J. Longhurst and N. Quirke, J. Chem. Phys. 124, 234708 (2006).

${ }^{18}$ J. Lefebvre, J. M. Fraser, Y. Homma, and P. Finnie, Appl. Phys. A: Mater. Sci. Process. 78, 1107 (2004).

${ }^{19}$ Y. Ohno, S. Iwasaki, Y. Murakami, S. Kishimoto, S. Maruyama, and T. Mizutani, Phys. Rev. B 73, 235427 (2006).

${ }^{20}$ H. Son, A. Reina, Ge. G. Samsonidze, R. Saito, A. Jorio, M. S. Dresselhaus, and Jing Kong, Phys. Rev. B 74, 073406 (2006).

${ }^{21}$ M. Y. Sfeir, T. Beetz, F. Wang, L. Huang, X. M. H. Huang, M. Huang, J. Hone, S. O’Brien, J. A. Misewich, T. F. Heinz, Lijun Wu, Yimei Zhu, and L. E. Brus, Science 312, 554 (2006).

${ }^{22}$ G. D. Mahan, Phys. Rev. B 65, 235402 (2002).

${ }^{23}$ A. Jorio, C. Fantini, M. A. Pimenta, R. B. Capaz, G. G. Samsonidze, G. Dresselhaus, M. S. Dresselhaus, J. Jiang, N. Kobayashi, A. Gruneis, and R. Saito, Phys. Rev. B 71, 075401 (2005).

${ }^{24}$ V. N. Popov and P. Lambin, Phys. Rev. B 73, 085407 (2006).

${ }^{25}$ C. Fantini, A. Jorio, A. P. Santos, V. S. T. Peressinotto, and M. A. Pimenta, Chem. Phys. Lett. 439, 138 (2007).

${ }^{26}$ U. H. F. Zimmerli, Nano Lett. 5, 1017 (2005).

${ }^{27}$ L. Grigorian, S. Colbern, I. O. Maciel, M. A. Pimenta, F. Plentz, and A. Jorio, Nanotechnology 18, 435705 (2007). 